\title{
Expression of oxidative stress and antioxidant defense genes in the kidney of inbred mice after intestinal ischemia and reperfusion ${ }^{1}$
}

\author{
Roberto Teruya ${ }^{\mathrm{I}}$, Adauto Tsutomu Ikejiri" ${ }^{\mathrm{II}}$, Frederico Somaio Neto"I, José Carlos Chaves ${ }^{\mathrm{III}}$, Paulo Roberto Bertoletto ${ }^{\mathrm{IV}}$, Murched \\ Omar Tahav, Djalma José Fagundes ${ }^{\mathrm{VI}}$
}

IFellow PhD degree in Surgery, Postgraduate Program in Interdisciplinary Surgical Sciences, Sao Paulo Federal University (UNIFESP). Affiliate Professor, Surgery Department, Mato Grosso do Sul Federal University (UFMS), Campo Grande-MS, Brazil. Intellectual, design and scientific content of the study, technical procedures, acquisition and interpretation of data, statistical analysis, manuscript writing, critical revision.

IIFellow Master degree in Surgery, Postgraduate Program in Interdisciplinary Surgical Sciences, UNIFESP, Sao Paulo-SP. Associate Professor, Medical School, Grande Dourados Federal University (UFGD), Dourados-MS, Brazil. Technical procedures, acquisition and interpretation of data, statistical analysis, manuscript writing.

IIIFellow PhD degree in Surgery, Postgraduate Program in Interdisciplinary Surgical Sciences, UNIFESP, Sao Paulo-SP. Associate Professor, Medical School, UFGD, Dourados-MS, Brazil. Technical procedures, acquisition and interpretation of data, statistical analysis, manuscript writing.

${ }^{\text {IV }} \mathrm{PhD}$, Associate Professor, Medical School, UFGD, Dourados-MS, Brazil. Technical procedures, acquisition and interpretation of data, statistical analysis, manuscript writing.

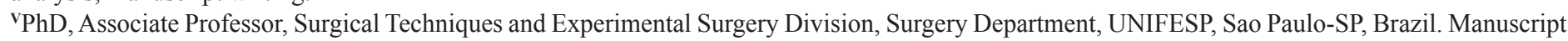
writing, critical revision.

vIPhD, Associate Professor, Surgical Techniques and Experimental Surgery Division, Surgery Department, UNIFESP, Sao Paulo-SP, Brazil. Conception and design of the study, manuscript writing,

critical revision.

\section{ABSTRACT}

PURPOSE: To determine the gene expressions profile related to the oxidative stress and the antioxidant response in the kidneys of mice subjected to intestinal ischemia and reperfusion.

METHODS: Twelve inbred mice (C57BL/6) were randomly assigned to one of two groups: the control group (CG) underwent anesthesia and was observed for $120 \mathrm{~min}$ and the ischemia/reperfusion group (IRG), animals were anesthetized and subjected to laparotomy and ischemia for 60 minutes followed by 60 minutes of reperfusion. The expressions of 84 genes from the kidney were determined by the Reverse Transcription qualitative Polymerase Chain Reaction (RT-qPCR). All genes that were up regulated by more than threefold using the algorithm $[2(\Delta \Delta \mathrm{Ct})]$ were considered statically significant $(\mathrm{p} \leq 0.05)$.

RESULTS: In the IRG group 29 (34.52\%) of 84 genes, were up regulated by more than threefold. The genes that were differentially up regulated in the glutathione peroxidase cluster (10 genes): were Gpx2 and Gpx7. The genes that were up regulated in the peroxidase cluster (16 genes) were following: Duox1, Epx, Lpo, Mpo, Ptgs2, Rag2, Serpinb1b, Tmod1 and Tpo. The genes that up regulated in the reactive oxygen species cluster (16 genes): I119, I122, Nos2, Nox1, Noxa1, Noxo1, Recq14 and Sod2. The genes that were up regulated in the oxidative stress cluster (22 genes) were: Mpp4, Nudt15, Upc3 and Xpa. The genes that were up regulated in the oxygen carriers cluster (12 genes) were: Hbq1, Mb, Ngb, Slc38al and Xirp1. The peroxiredoxins genes (10) showed no consistent differential regulation. CONCLUSION: The genes related to oxidative stress and antioxidant defense showed increased expression in renal tissue trigged intestinal ischemia and reperfusion.

Key words: Oxidative Stress. Gene Expression. Kidney. Reperfusion Injury. Mice 


\section{Introduction}

Ischemia-reperfusion (IR) injury occurs when a tissue is temporarily deprived of blood supply and the return of the circulation triggers an intense inflammatory response $\mathrm{e}^{1-3}$. Ischemia can be caused by either arterial or venous occlusion leading to blood stasis in the affected vascular bed ${ }^{3}$. Although oxygen is a critical substrate in the improvement of ischemia, paradoxically, it also functions as a deleterious metabolite during the reperfusion of previously ischemic tissues ${ }^{1-3}$.

Intestinal IR is a common clinical problem after small bowel transplantation, circulatory shock and strangulation ileus. IR causes intestinal dysfunction characterized by histological mucosa damage, decreased basal membrane integrity and impairment of barrier function ${ }^{1}$. The intestinal injuries triggered by ischemia also persist during reperfusion and involve activation of cytotoxic and inflammatory cascades and the expression or suppression of new gene products ${ }^{1-4}$. Although the molecular mechanisms underlying acute intestinal injury and repair after IR have not been completely elucidated, it is known that reactive oxygen species (ROS) play a role in acute intestinal injury after IR, in local tissue and in remote organs. Therefore, intestinal IR promotes inflammation and multiorgan failure $(\mathrm{MOF})^{3-6}$. Both, ROS and inflammatory cytokines play a role in kidney injury after IR intestinal ${ }^{6}$.

In the kidney the most prominent changes following IR are the swelling of endothelial cells and the destruction of mitochondrial cristae in the endothelial cells of glomerular capillaries and the epithelial tubular cells. Additionally, blood urea has been shown to increase as the inulin clearance decreases ${ }^{6}$.

Experiments with free radical scavengers have shown that DNA damage is dependent on ROS generation. Oxidative stress generated directly by ROS or through lipoperoxidation products, can initiate the activation of specific transcription factors and the expression of appropriate target genes in response to the injury ${ }^{1-6}$. Understanding the interrelationships of these genes is important for identifying potential targets for therapeutic intervention against intestinal $\mathrm{IR}^{5}$.

This study focused on analyzing gene expression associated with the oxidative stress and oxidative defense in kidney tissue after IR of small bowel. Herein, we have employed RT-qPCR (Reverse Transcriptase-quantitative Polymerase Chain Reaction) $)^{7}$ array technology which is a powerful tool to simultaneously analyze gene expression in the kidney. The objective was to determine the profile of gene expressions related to oxidative stress and antioxidant response in the kidneys of mice subjected to intestinal ischemia and reperfusion.

\section{Methods}

The experimental protocol (\#1598/08) was approved by the Ethics Committee of the Sao Paulo Federal University (UNIFESP), Brazil. Inbred mice (C57BL/6) were maintained according to the Guide for The Care and Use of Laboratory Animals (Institute for Laboratory Animal Research, 1996). The study was designed as a randomized controlled trial with a blinded assessment of the outcome.

Twelve male inbred C57BL/6 mice (Center for the Development of Experimental Models for Medicine and Biology - CEDEME-UNIFESP) that weighed from 30 to $35 \mathrm{~g}$ were housed under temperature and light controlled conditions. The animals had free access to water and standard pellet chow until 6 hours prior to the surgical procedures.

The animals were randomly assigned to one of two groups: the control group (CG) animals were anesthetized and received laparotomy and were then observed for $120 \mathrm{~min}$, while the ischemia/reperfusion group (IRG) animals were to anesthetized and then received laparotomy and $60 \mathrm{~min}$ of small bowel ischemia followed by $60 \mathrm{~min}$ of reperfusion.

\section{Anesthesia and surgical procedures}

After $6 \mathrm{~h}$ of fasting from a solid diet and $4 \mathrm{~h}$ from a liquid diet, the animals received a combination of $44 \mathrm{mg} / \mathrm{kg}$ of ketamine i.m, $2.5 \mathrm{mg} / \mathrm{kg}$ of xylazine i.m. and $0.75 \mathrm{mg} / \mathrm{kg}$ of acepromazine i.m. Anesthesia was maintained with an open oxygen mask. The animal body temperature was maintained at $37.8^{\circ} \mathrm{C}$ using a homoeothermic soft blanket. Under aseptic conditions, all 12 animals underwent a midline laparotomy. The superior mesenteric artery was carefully dissected and then occluded by a vascular clamp for 60 minutes. Ischemia was confirmed by observing the pale appearance of the clamped small bowel and the absence of beats in the mesenteric artery branches. After the clamp was removed, reperfusion was evaluated based on immediate color recovery and artery beats ( $\mathrm{x} 4$ magnification device). The surgical wounds remained covered with wet gauze wrappings throughout the experiment to minimize evaporative loss. After $120 \mathrm{~min}$ in the CG group and 60 min after the reperfusion in the IRG group, the surgical wounds were opened and samples were collected from the left kidney. The samples were harvested in the less than one and half minutes and the frozen in liquid nitrogen. After the samples were collected, the animals were sacrificed by decapitation. 


\section{$R N A$ preparation $(R T-q P C R)$}

$\mathrm{RT}^{2}$ Profiler ${ }^{\mathrm{TM}}$ PCR array from SA Biosciences (Frederick, Maryland; cat \# PAMM-065) was performed for mouse oxidative stress and antioxidant defense pathways according to the manufacturer's protocol. Briefly, total RNA was extracted from CG group and IRG group kidney tissues using Trizol reagent (Life Technologies, Grand Island, NY, USA). The RNA was then purified (Rneasy MiniKit Qiagen Co, USA). The concentration of total RNA samples was determined by spectrophotometry and the quality was assessed using analysis on a $2 \%$ agarose gel. One microgram of total RNA was used to make the first strand complementary DNA (cDNA) using a RT2 First Strand Kit (SA Biosciences). An equal amount of cDNA was mixed with SYBR Green (SA Biosciences Qiagen, Co) and dispensed in to each well of the PCR array plate containing the pre-dispensed gene-specific primer sets. The PCR was performed according to manufacturer's instructions. The PCR was performed in 96 well plates with 84 genes related to oxidative stress, five housekeeping genes (Actin B, Gapdh, Hsp90ab1, Hprt1, Gusb) were used for normalizing the PCR array data, and one negative control was used to monitor for genomic DNA contamination. The negative control primer set specifically detects non-transcribed, repetitive genomic DNA with a high level of sensitivity. The PCR array also contained three wells of reverse transcription controls (RTC) to verify the efficiency of the RT reaction. The qPCR assay specifically detects template synthesized from the first strand synthesis kit's built-in external RNA control. The replicate positive PCR controls (PPC) were used to check the efficiency of the polymerase chain reaction. These elements use a pre-dispensed artificial DNA sequence and the primer set that detects it. The two sets of replicate control wells (RTC and PPC) also test for inter-well and intra-plate consistency. The instrument's software (MxPro Equipment Real Time Systems, Stratagene, GE, Co) calculates the threshold cycle $(\mathrm{Ct})$ values for all the genes in the array. Finally, the software calculates fold changes in gene expression for pair wise comparison using the $\Delta \Delta \mathrm{Ct}$ method from the raw threshold cycle data. This method was used in our study to determine the relative expression levels of genes of interest for each sample. The data were stored in a spreadsheet for analysis using PCR Array Data Analysis v3.3 (SA Biosciences Qiagen, Co).

\section{Statistical analysis}

Gene expression data for each sample was evaluated in triplicate. The Student $t$ test $(p<0.05)$ was used to validate the homogeneity of the expression of each gene. For comparisons between the two groups, the computer program calculated the variation of cycle quantification $(\mathrm{Ct})$ in the study group compared to the quantification cycle $(\mathrm{Ct})$ in the control group. The data were expressed as in logarithmic base (2) and expression was calculated using the formula $2^{\wedge}(\Delta \Delta \mathrm{Ct})$. We considered genes with statistically significant changes expression and a threshold with more or less than a threefold up regulation or down regulation.

\section{Results}

The scatter plot (Figure 1) shows the up regulation and down regulation fold change between the test sample and controls in the 84 genes investigated.

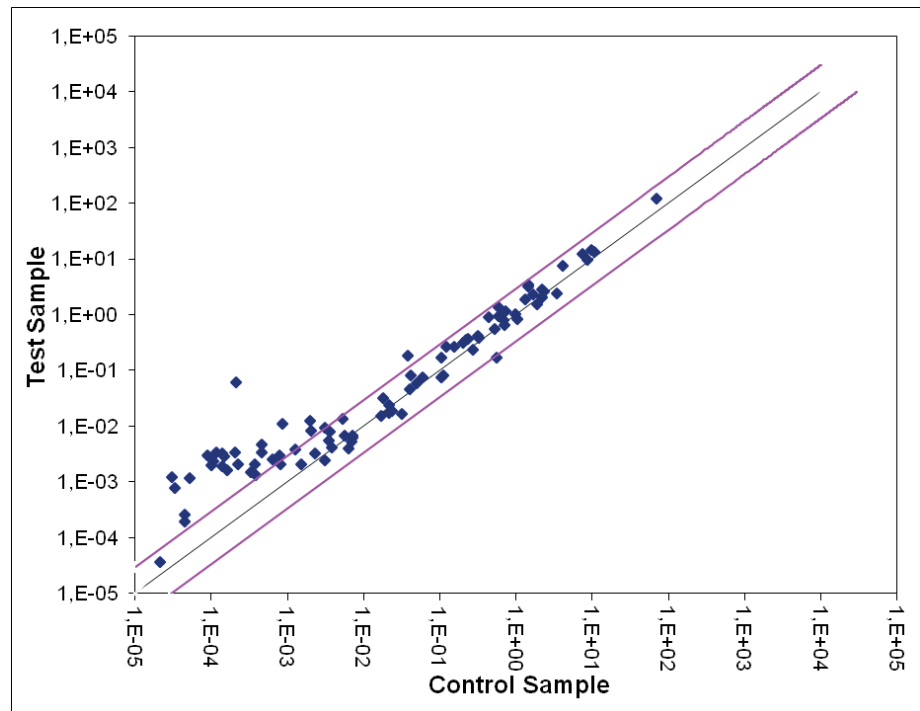

FIGURE 1 - Scatter plot allows the visualization of the up regulation and down regulation of all the genes whose expression was investigated. The black line indicates fold changes $\left[\left(2^{\wedge}\left(\Delta \Delta \mathrm{C}_{\mathrm{t}}\right)\right]\right.$ of 1 . The pink lines indicate a threefold-change in the gene expression threshold.

Eighty-four genes were examined in the kidneys of inbred mice affected by oxidative stress, following intestinal ischemia and reperfusion. Twenty-nine genes $(34.5 \%)$ were up-regulated and zero $(0 \%)$ were down-regulated. The gene identification abbreviation (alphabetical order), description, p-value and expression the threshold of gene with a more or less than threefold up regulation or down regulation is shown in Table 1. 
TABLE 1 - Distribution of 29 genes among the 84 genes investigated in the kidney with a threefold-change in the gene expression threshold in animals subjected to $60 \mathrm{~min}$ of ischemia of the small intestine and $60 \mathrm{~min}$ of reperfusion compared to the control group without ischemia/reperfusion $(*=$ significant $\mathrm{p}<0.05)$.

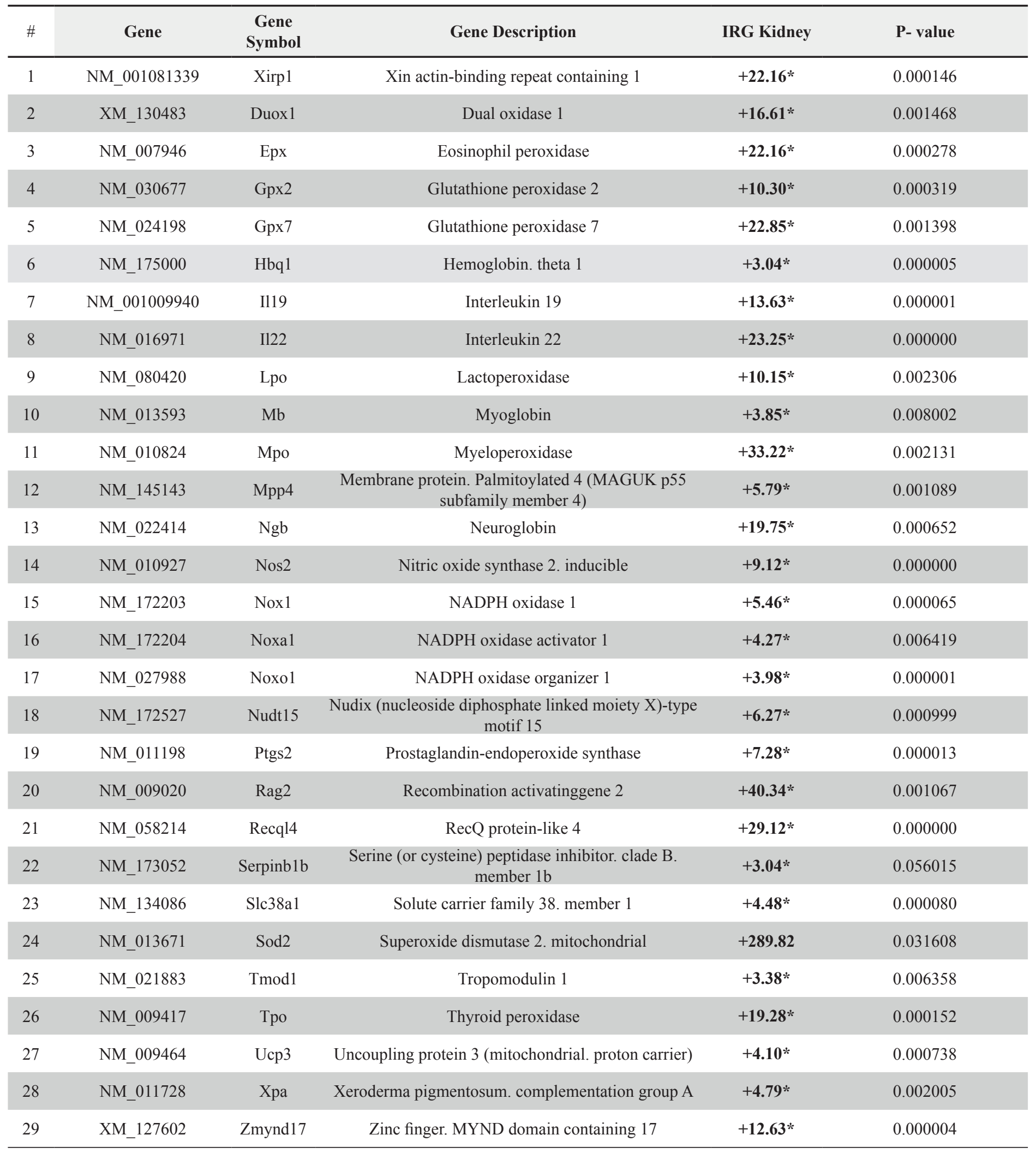


The six major functional gene clusters examined by the RT-qPCR kit were subjected to hierarchical clustering analysis using the $\left[\left(2^{\wedge}\left(\Delta \Delta \mathrm{C}_{\mathrm{t}}\right)\right]\right.$ method from the raw threshold cycle data (Table 2).

TABLE 2 - Distribution of the clusters and numbers of genes in the cluster, as well as the gene abbreviations and the respective threefold-change in up regulation of oxidative stress gene expression in the kidney of mice that was triggered by intestinal ischemia and reperfusion.

\begin{tabular}{|c|c|c|c|c|c|}
\hline \# & Cluster & No.genes & Genes & Up-regulated & $\mathrm{N} /(\%)$ \\
\hline 3 & Peroxidases (Pox) & 16 & $\begin{array}{c}\text { Aass, Apc, Cat, Ctsb, Duox1, Epx, Lpo, } \\
\text { Mpo, Ptgs1, Ptgs2, Rag2, RGD1560658 } \\
\text { (Serpinb1b), RGD1565187 (Kif9), Slc41a3, } \\
\text { Tmod1, Tpo. }\end{array}$ & $\begin{array}{l}\text { Duox1, Epx, Lpo, } \\
\text { Mpo, Ptgs2, Rag2, } \\
\text { Serpinb1b, Tmod1, } \\
\text { Tpo }\end{array}$ & $9(10.7 \%)$ \\
\hline 4 & $\begin{array}{l}\text { Reactive oxygen species } \\
\text { (Ros) }\end{array}$ & 16 & $\begin{array}{c}\text { Ccs, Cyba, Fmo2, I119, I122, Ncf2, Nos2, } \\
\text { Nox1, Nox4, Noxa1, Noxo1, Recq14, Scd1, } \\
\text { Sod1, Sod2, Sod3. }\end{array}$ & $\begin{array}{l}\text { Il19, Il22, Nos2, } \\
\text { Nox1, Noxa1, Noxo1, } \\
\text { Recq14, Sod2 }\end{array}$ & $8(50.0 \%)$ \\
\hline \multirow[t]{2}{*}{6} & $\begin{array}{c}\text { Oxygen transporters } \\
\text { (Trox) }\end{array}$ & 12 & $\begin{array}{c}\text { Aqr, LOC367198 (Atr), Cygb, Dnm2, Fancc, } \\
\text { Hbq1, Slb (Ift172), Mb, Ngb, Slc38a1, Vim, } \\
\text { Xirp1. }\end{array}$ & $\begin{array}{l}\mathrm{Hbq} 1, \mathrm{Mb}, \mathrm{Ngb} \\
\text { Slc38a1, Xirp1 }\end{array}$ & $5(41.6 \%)$ \\
\hline & TOTAL & $\begin{array}{c}84 \\
(100 \%)\end{array}$ & & & $29(34.52 \%)$ \\
\hline
\end{tabular}

\section{Discussion}

Our data show that intestinal ischemia followed by reperfusion promoted a statistically significant increased expression in 29 (34.5\%) of 84 genes in the kidney related to the oxidative stress and antioxidant defense (Table 1 and Figure 1). The findings suggest that IR plays a pivotal role in the expression of genes encoding proteins involved in the generation and modulation of ROS.

Previous reports have shown that the kidney is injured under conditions of ischemia triggered in remote organs. The main morphological changes that occur in the kidney are swelling of endothelial cells, destruction of mitochondrial cristae in the endothelial cells of the glomerular capillaries and epithelial tubular cells. Additionally, functional changes are caused by increased blood urea nitrogen and decreased inulin clearance $e^{6,8}$.

The initial lesion in intestinal tissue promotes the recruitment of cells such as neutrophils and the release of various mediators including complement, vasoactive mediators, eicosanoids, nitric oxide and inflammatory cytokines that can act in remote organs ${ }^{3,4,9}$. The molecular mechanisms underlying acute intestinal injury after IR have not been fully elucidated. However, oxidative stress may be the most relevant, not only locally but also in remote organs, such as the kidney.

Studies have shown proteins that function together in a 
pathway or structural complex are likely to evolve in a correlated manner. The proteins that have matching profiles or similar trends are functionally linked ${ }^{10}$. Determining protein functions from genomic sequences is a key challenge. Therefore, we analyzed our data by grouping genes according to their similarity based on known activity in biochemical pathways. Such clusters are readily accessible using universal primers and are typically present in high copy number in the cell. The genes in a cluster, often exhibit sequence divergence that allows for the design of specific primers. This study used our current knowledge to elucidate this relationship ${ }^{1}$.

The data collected in this study support the conclusion that intestinal ischemia followed by reperfusion is a phenomenon that is closely related to reactive oxygen species. The local changes in the bowel promoted a humoral and cellular spread to the blood stream, which affects the expression of genes related to the generation and modulation of ROS in renal tissue.

There are currently no reports investigating the association of gene expression with intestinal ischemia and renal remote injury. Thus, this study was based on the available literature on the expression of genes. Grouping the genes into clusters enabled the detailed analysis of the relationships between genes that encode proteins with a deleterious or protective role in renal tissue.

Glutathione peroxidases play a pivotal role in the protection against the noxious activity of ROS. They are present in the most animal cells and promote the reduction of lipid hydroperoxides in the alcohols and oxidants of hydrogen peroxides $\left(\mathrm{H}_{2} \mathrm{O}_{2}\right)$ in free water by removing the ROS from the interior of the cells. The rate of reduction of glutathione to the oxidizing form can be used as a monitor of cellular toxicity ${ }^{11}$. Our results show that only the expression two isoforms (Gpx2 and Gpx7) are significantly increased. Gpx2 is a selenium dependent enzyme that is located in the gastrointestinal tract that responds to oxidative stress $^{12}$, whereas Gpx7 is associated with decreased oxidative stress metabolism of polyunsaturated fats ${ }^{11,13}$.

The peroxiredoxins represent an important family of proteins that are related to the thiol-peroxidases and are present in all known organisms. These proteins have an antioxidant function and can, reduce $\mathrm{H}_{2} \mathrm{O}_{2}$, organic peroxides and peroxinitrite to water, alcohol and nitrite. The peroxiredoxinas are associated with both antioxidant protection and redox signaling ${ }^{14,15}$. Our results from the gene expression analysis in kidney tissue were within the normal range of thresholds in all study groups. This indicates that the metabolic pathways for reducing oxidative stress do not require the synthesis of the proteins encoded by these genes.

Peroxidases are a large family of catalyst enzymes that use hydrogen peroxide as a substrates. However, the enzymes can also use of organic hydroperoxides as substrates. The enzymes play an important role in modulating oxidative stress ${ }^{17}$. Of the sixteen peroxidases examined nine $(56.2 \%)$ were up-regulated by more than threefold (Duox1, Epx, Lpo, Mpo, Ptgs2, Rag2, Serpinb1b, Tmod1, Tpo). Each of these genes has a specific metabolic pathway and performs a variable action in different tissues. The scope of our research is aimed at mapping the overall expression of these genes and our results open new areas of investigation for upcoming studies.

Most of the oxygen consumed by aerobic organisms is reduced to water. A significant proportion of molecular oxygen ions are converted to superoxide anion radicals such as oxygen ions and peroxides, which are highly chemically reactive due to the presence of unpaired electrons producing the ROS ${ }^{1,17}$. These ROS can increase dramatically in hypoxic conditions and are activated as endogenous defense mechanisms to maintain the cellular homeostasis. The expression of genes critical to tissue oxygen consumption may signal either favorable effects or worsening tissue conditions ${ }^{17}$. The effect of ROS in cell metabolism has been well documented in various organs and tissues in many species. There are reports of their function directly in the process of apoptosis in response to the expression defense genes ${ }^{1,3,17}$. In our study, eight $(50 \%)$ of the genes in the ROS cluster surveyed were above the threefold up regulation threshold (I119, I122, Nos2, Nox1, Noxa1, Noxo1, Recq14, Sod2). Most of these genes are known to be involved in antioxidant defense. The mapping of gene expression allows these genes to be further investigated in future research.

Oxidative stress results from the imbalance between the production of ROS and the ability of natural biological defense mechanisms to prevent or reduce harmful effects to cells by repairing the damage caused by oxidative stress ${ }^{17,18}$. The toxic effects are caused by the production of oxygen free radicals that damage cellular components (protein, lipids and DNA) in situations where increased levels of oxidative stress exceed physiological barriers of defense. The severity of the stress is related to the strength of that stimulus and the time of action of that stimulus. The results of the imbalance can range from repairing damage to reverse the actions induced cell death by apoptosis or necrosis ${ }^{17-19}$. The analysis of 22 genes involved in oxidative stress cluster of showed that five $(22.7 \%)$ of the genes were upregulated by threefold (Mpp4, Nudt15, Ucp3, Xpa and Zmynd17). Quantitatively, the number of up-regulated genes was relatively small and the threefold change in the gene expression threshold was lower than for other genes in the ROS family. It is possible 
that ischemia and reperfusion is not a consistent stimulate or of oxidative stress in the kidneys.

The cluster of oxygen transporters are formed by genes encoding various chemical classes, and the various stages of transport of the gas involves several steps. Many parts of the pathway are unclear and there is no consensus regarding the understanding of metabolic pathways and the importance of each step. The transport of oxygen is related to the ability to link hemoglobin and erythrocytes transported to the capillaries. Inside the red blood cell hemoglobin molecules undergo performance changes to meet specific physiological requirements ${ }^{1}$. The capacity of oxygen that can be absorbed by cells is dependent on carriers and pores that may open and close according to intra and extracellular stimuli. In tissues, modulation mechanisms are used to perform a variety of other features in relation to the basic function of oxygen transport ${ }^{1,20,21}$. Our results show that five (41.2\%) oxygen transporter genes were up-regulated threefold (Hbq1, Mb, Ngb, Slc38a1, Xirp1). The regulation of oxygen transport to the kidney cells is affected by the stimulus at a distance when triggered by intestinal ischemia. Additional research to elucidate this process should be based on our expression mapping data.

Using an RT-qPCR array containing, 84 genes related to oxidative stress and antioxidant defense allowed us to evaluate the stimulus of ischemia and reperfusion on renal tissue in inbred mice. The results indicate a clear expression profile of these genes. A literature review showed no other work involving this type of model. Our mapping strategy opens the possibility of further studies to evaluate each of these genes or their clusters. Additionally, future studies will be able to compare of the use of different drugs and antioxidants, scavengers of ROS, ischemic preconditioning procedures and hyperbaric oxygen and other procedures that are used to mitigate or eliminate the effects of intestinal ischemia and reperfusion injury on the kidney tissue. This report may also facilitate the identification of conditions to monitor intestinal transplants.

\section{Conclusion}

The genes related to oxidative stress and antioxidant defense showed increased expression in renal tissue triggered by intestinal ischemia and reperfusion.

\section{References}

1. Bertoletto PR, Ikejiri AT, Somaio Neto F, Chaves JC, Teruya R, Bertoletto ER, Taha MO, Fagundes DJ. Oxidative stress gene expression. Profile in inbred mouse after ischemia/reperfusion small bowel injury. Acta Cir Bras. 2012;27(11):773-82.

2. Moore-Olufemi SD, Kozar RA, Moore FA, Sato N, Hassoun HT, Cox Jr CS, Kone BC. Ischemic preconditioning protects against gut dysfunction and mucosal injury after ischemia/reperfusion injury. Sock. 2005;23(3):258-63.

3. Gilany K, Vafakhah M. Hypoxia: a review. J Paramed Sci. 2010;1(2):43-60.

4. Milano PM, Douillet CD, Riesenman PJ, Robinson WP, Beidler SK, Zarzaur BL, Rich PB. Intestinal Ischemia-reperfusion injury alters purinergic receptor expression in clinically relevant extra-intestinal organs. J Surg Res. 2008;145(2):272-8.

5. Mallick IH, Yang W, Winslet MC, Seifalian AM. Ischemiareperfusion injuries of the intestine and protective strategies against injury. Dig Dis Sci. 2004;49(9):1359-77.

6. Ogura J, Kobayashi M, Itagaki S, Hirano T, Iseki K. Alteration of Mrp2 and P-gp expression, including expression in remote organs after intestinal ischemia-reperfusion. Life Sci. 2008;82:1242-8.

7. Deepak SA, Kottapalli KR, Rakwal R, Oros G, Rangappa KS, Iwahashi H, Masuo Y, Agrawal GK. Real-time PCR: revolutionizing detection and expression analysis of genes. Curr Genomics. 2007;8:234-51.

8. Mutlu G, Abbasoglu L, Dogru-Abbasoglu S, Solkoglu S Bulut. Morphologic changes and lipid peroxidation in renal tissues of young rats following intestinal ischemia-reperfusion. Pediatr Surg Int. 2002;18:337-40

9. Sizlan A, Guven A, Uysal B, Yanarates O, Atim A, Oztas E, Cosar A, Korkmaz A. Proanthocyanidin protects intestine and remote organs against mesenteric ischemia/reperfusion injury. World J Surg. 2009;33:1384-91.

10. Pellegrini M, Marcotte ED, Thompson MJ, Eisenberg D, Yeates TO. Assigning protein functions by comparative genome analysis: protein phylogenetic profiles. Proc Natl Acad Sci USA. 1999;96(8):4285-8.

11. Toppo S, Vanin S, Bosello V, Tosatto SCE. Evolutionary and structural insights into the multifaceted Glutationa peroxidases (Gpx) superfamily. Antioxid Redox Signal. 2008;10(8):2008-57.

12. Wang J, Chen L, Li P, Li X, Zhou H, Wang F, Li D, Yin Y, Wu G. Gene expression is altered in piglet small intestine by weaning and dietary glutamine J Nutr. 2008;138:1025-32.

13. Talmud PJ, Drenos F, Shah S, Sah T, Palmen J, Verzilli C, Gaunt TR, Pallas J, Lovering R, Li K, Casas JP, Sofat R, Kumari M, Rodrigurez S. Johnson T, Newhouse J, Dominiczak A, Samani NJ, Caulfield M, Sever P, Stanton A, Shields DC. Gene-centric association signal for lipid and apolipoproteins identified via the humana cvd beadchip. Am J Hum Gen. 2009;85:628-42.

14. Hall A, Karplus PA, Poole LB. Typical 2-Cys perodoxiredoxins: structures, mechanisms and functions. Febs J. 2009;276(9):2469-77.

15. Ha B, Kim E, Kim J, Lee HN, Lee KO, Lee SY Jnag HH. Human peroxiredoxin 1 modulates TGF- $\beta 1$-induced epithelialmesenchymal transition through its peroxidase activity. Biochem Biophys Res Commun. 2012;421:33-7.

16. Ishibashi $\mathrm{N}$, Weisbrot-LefkowitzM, Reuhl $\mathrm{K}$, Inouye $\mathrm{M}$, Mirochnitchenko O. Modulation of chemokine expression during ischemia/reperfusion in transgenic mice overproducing human glutathione peroxidases. J Immunol. 1999;163:5666-77.

17. Ferreira ALA, Matsubara LS. Radicais livres: conceitos, doenças relacionadas, sistema de defesa e estresse oxidativo. Rev Assoc Med Bras. 1997;43(1):61-8.

18. Valko M, Leibfritz D, Moncol J, Cronin MTD, Mazur M, Telser J. Free radicals and antioxidants in normal physiological functions and human disease. Int Biochem Cell Biol. 2007;39:44-84.

19. Lee Y, Shacter E. Oxidative stress inhibits apoptosis in human lymphoma cells. J Biol Chem. 1999;274:19792-8. 
20. De Rosa MC, Carelli Alinovi C, Galtieri A, Russo A, Giardina B. Allosteric properties of hemoglobin and the plasma membrane of the erythrocyte: new insights in gas transport and metabolic modulation. IUBMB Life. 2008;60(2):87-93.

21. Nagao S, Asami O, Yasui H, Hirota S. Efficient reduction of Cys 110 thiyol radical by glutathione in human myoglobin. Biochim Biophys Acta. 2011;1844:480-6.

\section{Acknowledgments}

To Operative Technique and Experimental Surgery Division, Department of Surgery and Molecular Gynecology Facilities and UNIFESP; the biologist, Fellow PhD degree, Molecular Gynecology Postgraduate Program, UNIFESP, Marcos Azevedo Junior for the gene expression technology and adviser support; Associate Professor, Department of Gynecology and Head of Molecular Gynecology Facilities, UNIFESP, Ismael Dale Cotrin Guerreiro da Silva for support with the analysis of the gene expression data and Maria do Carmo Negrini Fagundes for assistance with translation and formatting of the article. Financial support was partially provided by Grande Dourados Federal University.

\author{
Correspondence: \\ Roberto Teruya \\ Rua Aluizio de Azevedo, 1330/204 Bloco F \\ 79004-050 Campo Grande - MS Brasil \\ wakateteruya@uol.com.br \\ Tel.: (55 67)3341-3069
}

Received: Aug 26, 2013

Review: Oct 25, 2013

Accepted: Nov 26, 2013

Conflict of interest: none

Financial source: none

${ }^{1}$ Research performed at Laboratory of Experimental Surgery Department, Medical School, Grande Dourados Federal University (UFGD), Dourados-MS, Brazil. Part of PhD degree thesis, Postgraduate Program in Interdisciplinary Surgical Sciences, UNIFESP. Tutor: Djalma José Fagundes. 